\title{
Surface groups acting on CAT $(-1)$ spaces
}

\author{
Georgios Daskalopoulos ${ }^{1}$ \\ Brown University \\ daskal@math . brown . edu \\ Chikako Mese $^{2}$ \\ Johns Hopkins University \\ cmese@math. jhu .edu \\ Andrew Sanders ${ }^{3}$ \\ Heidelberg University \\ asanders@mathi . uni-heidelberg.de \\ and \\ Alina Vdovina ${ }^{4}$ \\ Heidelberg University \\ Newcastle University \\ Alina.Vdovina@newcastle.ac.uk
}

\begin{abstract}
Harmonic map theory is used to show that a convex cocompact surface group action on a CAT $(-1)$ metric space fixes a convex copy of the hyperbolic plane (i.e. the action is Fuchsian) if and only if the Hausdorff dimension of the limit set of the action is equal to 1. This provides another proof of a result of Bonk and Kleiner. More generally, we show that the limit set of every convex cocompact surface group action on a CAT $(-1)$ space has Hausdorff dimension $\geq 1$ where the inequality is strict unless the action is Fuchsian.
\end{abstract}

\footnotetext{
${ }^{1}$ supported by research grant NSF DMS-1608764

${ }^{2}$ supported by research grant NSF DMS-1406332

${ }^{3}$ supported by research grants NSF DMS-1304006, DMS-1107452, DMS-1107263 and DMS-1107367 "RNMS: Geometric structures And Representation varieties" (the GEAR Network)

${ }^{4}$ supported by EPSRC research grant EP/K016687/1
} 


\section{Introduction}

The relationship between dynamical properties of discrete group actions on metric spaces and rigidity theorems has a rich history: a prototypical result due to Bowen [Bow] states that the Hausdorff dimension of the limit set of a quasi-Fuchsian surface group $\Gamma$ acting on hyperbolic 3 -space is equal to 1 if and only if $\Gamma$ restricts to an isometric action on hyperbolic 2 -space ( $\Gamma$ is Fuchsian).

A generalization of this result to surface group actions on $\mathrm{CAT}(-1)$ metric spaces was originally conjectured by Bourdon [Bou]. It was later verified by Bonk and Kleiner [BK] where they prove a more general statement about quasi-convex actions.

In the special case when the space is a smooth Riemannian manifold with pinched negative sectional curvature, a different proof of this result was found by Deroin and Tholozan [DT] and Sanders [San] utilizing equivariant harmonic maps and an inequality which compared curvature quantities of the harmonic map with the Hausdorff dimension of the limit set. Here, we extend these techniques to the general CAT $(-1)$ setting using the harmonic map theory which has been developed by Koraveer-Schoen [KS1, KS2] and by Mese [Mes] to give a new proof of the original conjecture of Bourdon.

The main theorem of this paper is the following:

Theorem 1 Given a convex cocompact action $\rho: \pi_{1}(S) \rightarrow \operatorname{Isom}(X)$ on a $C A T(-1)$ metric space $X$ by the fundamental group of a closed, connected oriented surface $S$ with genus $>1$ such that $\operatorname{dim}_{\mathcal{H}}(\Lambda)=1$, there exists a hyperbolic metric $h$ on the universal cover $\tilde{S}$ of $S$ such that the unique $\rho$ equivariant harmonic map $\tilde{u}: \mathbb{H}^{2}=(\tilde{S}, h) \rightarrow X$ is a totally geodesic map and an isometric embedding.

In the above theorem, $\Lambda$ is the limit set of this action and the Hausdorff dimension is computed with respect to the distance $d_{p}(\xi, \eta)=e^{-(\xi \mid \eta)_{p}}$ where $p \in X$ and $\xi, \eta \in \partial_{\infty} X$. Finally, the expression $(\xi, \eta)_{p}$ is the Gromov product.

In the course of proving Theorem 1, we also prove the following.

Theorem 2 Given a convex cocompact action $\rho: \pi_{1}(S) \rightarrow \operatorname{Isom}(X)$ on a $C A T(-1)$ metric space $X$ by the fundamental group of a closed, connected oriented surface $S$ with genus $>1$, there exists a hyperbolic metric $h$ on the universal cover $\tilde{S}$ of $S$ and a contracting $\rho$-equivariant conformal harmonic map $\tilde{u}: \mathbb{H}^{2}=(\tilde{S}, h) \rightarrow X$. It is strictly contracting unless $\tilde{u}$ is totally geodesic and an isometric embedding of $\mathbb{H}^{2}$. 
In particular, Theorem 2 asserts that every representation $\rho$ of a closed surface with genus $>1$ is dominated by a Fuchsian representation. As noted by Deroin and Tholozan, the existence of a contracting map has some interesting consequences $[\mathrm{DT}]$.

In the Riemannian setting, Theorem 1 and Theorem 2 were proved by Deroin and Tholozan. Sanders [San] used a slightly different approach to prove Theorem 1. In the CAT $(-1)$ setting, Mese [Mes] established the existence of a metric (of low regularity) on $S$ for which the associated equivariant harmonic map is conformal. Moreover, where it makes sense, this metric is the pullback metric of the associated harmonic map. The main contribution of this paper is a comparison result (see Lemma 16) which allows us to trade this irregular metric for a smooth metric in the same conformal class. Using this smooth metric, we apply the maximum principle to prove Theorem 2 from which Theorem 1 follows. Theorem 1 immediately yields a new proof of the conjecture of Bourdon [Bou]:

Corollary 3 A convex cocompact isometric action $\rho: \pi_{1}(S) \rightarrow \operatorname{Isom}(X)$ on a CAT(-1) space $X$ by the fundamental group of a closed, connected oriented surface $S$ with genus $>1$ fixes a convex copy of $\mathbb{H}^{2}$ if and only if $\operatorname{dim}_{\mathcal{H}}(\Lambda)=1$.

More generally, Theorem 2 implies the following

Corollary 4 If $\rho: \pi_{1}(S) \rightarrow$ Isom $(X)$ is a convex cocompact isometric action on a $C A T(-1)$ space $X$ by the fundamental group of a closed, connected oriented surface $S$ with genus $>1$, then $\operatorname{dim}_{\mathcal{H}}(\Lambda) \geq 1$.

The paper is organized as follows. A brief preliminary section settles notation and states the fundamental existence result for equivariant harmonic maps of surfaces to CAT $(-1)$ metric spaces. Section 3 establishes the primary technical result alluded to in the introduction which replaces a conformal metric of low regularity with a comparable smooth Riemannian metric. Finally, Section 4 completes the proof of Theorem 1 and Theorem 2 from which Collorary 3 and Corollary 4 immediately follow.

Acknowledgements. The use of the maximum principle to prove our main results has its origin in [DT]. The original version of this manuscript followed the approach used by Sanders [San] instead. We thank the referee for pointing out $[\mathrm{DT}]$ and the resulting simpler argument now used in this paper. 


\section{Preliminaries}

Throughout this paper, $S$ denotes a closed, connected oriented surface of genus $>1, \tilde{S}$ its universal cover, and $\mathcal{T}$ the Teichmüller space of $S$. Furthermore, $X$ denotes a $\mathrm{CAT}(-1)$ space, $\operatorname{Isom}(X)$ the group of isometries of $X$ and $\rho: \pi_{1}(S) \rightarrow \operatorname{Isom}(X)$ a representation. We will identify an element of $\pi_{1}(S)$ as a deck transformation of $\tilde{S}$ and an element $h \in \mathcal{T}$ as an equivariant hyperbolic metric on $\tilde{S}$ (via uniformization).

Definition 5 Given a representation $\rho: \pi_{1}(S) \rightarrow \operatorname{Isom}(X)$, a map $\tilde{u}: \tilde{S} \rightarrow$ $X$ is said to be $\rho$-equivariant if

$$
\tilde{u}(\gamma x)=\rho(\gamma) \tilde{u}(x), \quad \forall x \in \tilde{S}, \gamma \in \pi_{1}(S) .
$$

Definition 6 A discrete subgroup $\Gamma$ of $\operatorname{Isom}(X)$ is said to be convex cocompact if there exists a geodesically convex, $\Gamma$-invariant subset of $X$ upon which $\Gamma$ acts cocompactly. A representation $\rho: \pi_{1}(S) \rightarrow \operatorname{Isom}(X)$ is said to be convex cocompact if $\Gamma=\rho\left(\pi_{1}(S)\right)$ is convex cocompact.

Definition 7 Given a hyperbolic metric $h \in \mathcal{T}$, the energy of a $\rho$-equivariant $\operatorname{map} \tilde{u}:(\tilde{S}, h)=\mathbb{H}^{2} \rightarrow X$ is

$$
E_{h}^{\tilde{u}}=\int_{F}|\nabla \tilde{u}|^{2} d h
$$

where $F \subset \mathbb{H}^{2}$ is a fundamental domain for the action of $\pi_{1}(S),|\nabla \tilde{u}|^{2}$ is the energy density function of $\tilde{u}$ as defined in [KS1], and $d h$ is the volume form associated with the metric $h$. Furthermore, we will denote

$$
E_{h}^{\tilde{u}}[U]=\int_{U}|\nabla \tilde{u}|^{2} d h
$$

for any measurable set $U \subset \mathbb{H}$.

Definition 8 Given a hyperbolic metric $h \in \mathcal{T}$, a finite energy map $\tilde{u}$ : $(\tilde{S}, h)=\mathbb{H}^{2} \rightarrow X$ is said to be harmonic if $E_{h}^{\tilde{u}}[\Omega] \leq E_{h}^{\tilde{v}}[\Omega]$ for any bounded Lipschitz domain $\Omega \subset \tilde{S}$ and any finite energy map $\tilde{v}: \Omega \rightarrow X$ with same boundary values as $\tilde{u}$.

Theorem 9 (cf. [KS1], [KS2]) Given a convex cocompact representation $\rho: \pi_{1}(S) \rightarrow X$ and a hyperbolic metric $h \in \mathcal{T}$, there exists a unique $\rho$ equivariant harmonic map $\tilde{u}:(\tilde{S}, h)=\mathbb{H}^{2} \rightarrow X$. Furthermore, $\tilde{u}$ is locally Lipschitz continuous. 


\section{Conformal Harmonic Maps}

Definition 10 Given a finite energy map $\tilde{u}: \Sigma \rightarrow X$ from a Riemann surface, let $\pi$ be the pullback inner product structure defined in [KS1] Theorem 2.3.2. If $z=x+i y$ is a local conformal coordinate on $\Sigma$ and $\left\{\frac{\partial}{\partial x}, \frac{\partial}{\partial y}\right\}$ are the coordinate vector fields, we obtain the locally integrable functions

$$
\left|\frac{\partial \tilde{u}}{\partial x}\right|^{2}:=\pi\left(\frac{\partial}{\partial x}, \frac{\partial}{\partial x}\right), \quad\left|\frac{\partial \tilde{u}}{\partial y}\right|^{2}:=\pi\left(\frac{\partial}{\partial y}, \frac{\partial}{\partial y}\right)
$$

and

$$
<\frac{\partial \tilde{u}}{\partial x}, \frac{\partial \tilde{u}}{\partial y}>:=\pi\left(\frac{\partial}{\partial x}, \frac{\partial}{\partial y}\right)
$$

Definition 11 A finite energy map $\tilde{u}: \Sigma \rightarrow X$ from a Riemann surface is said to be conformal if

$$
\left|\frac{\partial \tilde{u}}{\partial x}\right|^{2}=\left|\frac{\partial \tilde{u}}{\partial y}\right|^{2} \text { and }<\frac{\partial \tilde{u}}{\partial x}, \frac{\partial \tilde{u}}{\partial y}>=0
$$

where $(x, y)$ are local conformal coordinates of $\Sigma$. The local conformal factor of $\tilde{u}$ is the locally integrable function

$$
\lambda=\left|\frac{\partial \tilde{u}}{\partial x}\right|^{2}
$$

The next result is well-known. When the target space is a Riemannian manifold, it is due to Schoen and Yau (cf. [SY]). For the singular targets considered here, their argument goes through almost verbatim. See for example $[\mathrm{GW}]$.

Proposition 12 Given a convex cocompact representation $\rho: \pi_{1}(S) \rightarrow$ $\operatorname{Isom}(X)$, there exists $h \in \mathcal{T}$ and a $\rho$-equivariant conformal harmonic map $\tilde{u}: \mathbb{H}^{2}=(\tilde{S}, h) \rightarrow X$.

The following theorem is a summary of the results contained in [Mes].

Theorem 13 ( [Mes]) Given a conformal harmonic map $\tilde{u}: \Sigma \rightarrow X$ from a Riemann surface, the local conformal factor $\lambda$ satisfies the following properties: 
(i) $\lambda \in H_{l o c}^{1}(U)$,

(ii) $\log \lambda \in W_{l o c}^{1,1}(U)$,

(iii) $\lambda$ satisfies the weak differential inequality,

$$
\int_{U}(\triangle \varphi) \log \lambda d x d y \geq 2 \int_{U} \varphi \lambda d x d y, \quad \forall \varphi \in C_{c}^{\infty}(U), \varphi \geq 0
$$

where $\triangle$ is the Euclidean Laplacian in coordinates $(x, y)$ and

(iv) The zero set of $\lambda$ is of Hausdorff dimension zero; i.e.

$$
\operatorname{dim}_{\mathcal{H}}(\mathcal{D})=0 \quad \text { where } \quad \mathcal{D}=\{z=(x, y) \in U: \lambda(z)=0\} .
$$

In (iv), we let $\lambda$ be the representative function in the $L^{1}$-class defined everywhere by

$$
\lambda\left(z_{0}\right)=\lim _{r \rightarrow 0} \frac{1}{\pi r^{2}} \int_{D_{r}\left(z_{0}\right)} \lambda(z) d x d y .
$$

Definition 14 Given a conformal harmonic map $\tilde{u}: \Sigma \rightarrow X$ from a Riemann surface, the pullback metric of $\tilde{u}$ is the equivariant (possibly degenerate) twoform $G$ defined locally by

$$
G=\lambda\left(d x^{2}+d y^{2}\right)
$$

where $\lambda$ is the local conformal factor of $\tilde{u}$.

The weak differential inequality (1) allows us to use mollification (i.e. convolution) to construct smooth approximating metrics of (2). Furthermore, by mollifying with respect to a smooth radial function with compact support, we preserve the property of having curvature $\geq 1$ (which is true for (2) in the weak sense by (1)). More precisely, the following statement is proven is [Mes] Section 6:

Theorem 15 ( [Mes]) Let $u: \Sigma \rightarrow X$ be a conformal harmonic map from a Riemann surface and $\lambda$ be the local conformal factor in a coordinate neighborhood $U \subset \Sigma$. If $\lambda^{\sigma}=e^{(\log \lambda)_{\sigma}}$ where $(\log \lambda)_{\sigma}$ is a symmetric mollification (i.e. convolution by a radially symmetric function with compact support in a local coordinate) of $\log \lambda$ in a local coordinate neighborhood $U$, then

$$
\triangle \log \lambda^{\sigma} \geq 2 \lambda^{\sigma}
$$

and

$$
\lambda^{\sigma} \geq \lambda
$$


For the purposes of this paper, we need the following global analogue of Theorem 15.

Lemma 16 Given a convex cocompact representation $\rho: \pi_{1}(S) \rightarrow \operatorname{Isom}(X)$, $h \in \mathcal{T}$ a hyperbolic metric, a $\rho$-equivariant conformal harmonic map $\tilde{u}$ : $\mathbb{H}^{2}=(\tilde{S}, h) \rightarrow X$ and $G$ defined by (2), there exists a smooth equivariant conformally equivalent metric $G^{\sigma}$ on $\tilde{S}$ satisfying the following properties:

(i) The Gaussian curvature $K^{\sigma}$ of $G^{\sigma}$ satisfies $K^{\sigma} \leq-1$.

(ii) If $K^{\sigma}=-1$ at one point, then $G=G^{\sigma}=h$.

(iii) The metrics satisfy the inequality

$$
G(V, V) \leq G^{\sigma}(V, V), \quad \text { a.e. } q \in \tilde{S}, \forall V \in T_{q} \tilde{S}
$$

Proof. For $\kappa>0$ to be chosen later (cf. (15) below), let $g=\kappa^{-1} h$; thus $g$ is a metric on $\tilde{S}$ with constant Gaussian curvature $-\kappa$. Define the $\rho$-invariant locally integrable function

$$
f=\frac{G}{g}: \tilde{S} \rightarrow[0, \infty)
$$

In order to construct a smooth metric $G_{\sigma}$, we will use a convolution of log $f$ by a smooth radial function with compact support to define a smooth function on $\tilde{S}$.

Let $D=\left\{(x, y) \in \mathbb{R}^{2}: r=\sqrt{x^{2}+y^{2}}<1\right\}$ and consider the scaled Poincaré disk

$$
\left(D, \omega(r)\left(d x^{2}+d y^{2}\right)\right)
$$

where

$$
\omega(r)=\kappa^{-1} \frac{4}{\left(1-r^{2}\right)^{2}}
$$

In particular,

$$
\triangle \log \omega=2 \kappa \omega .
$$

Fix $q \in \tilde{S}$ and identify $(\tilde{S}, g)$ to the scaled Poincaré disk with $q$ corresponding to the origin. For clarity, we will refer to the coordinates $(x, y)$ as the Poincaré coordinates centered at $q$. Fix $\sigma>0$ and consider a smooth radially symmetric function $\varphi_{\sigma}$ with support contained in $D_{\sigma}(0):=\{(x, y)$ : $r<\sigma\} \subset D$ and the measure $d \nu_{q}=\varphi_{\sigma}(r) d x d y$ on $D$ supported in $D_{\sigma}(0)$. 
Via the identification of $\tilde{S}$ to $D$, we can consider $d \nu_{q}$ as a measure defined on $\tilde{S}$. Furthermore, by multiplying $\varphi_{\sigma}$ by an appropriate constant if necessary, we can assume that

$$
\int_{\tilde{S}} d \nu_{q}(p)=\int_{D_{\sigma}(0)} \varphi_{\sigma}(r) d x d y=1
$$

If $d g$ is the volume measure associated to the metric $g$, then we can write $d g$ in the Poincaré coordinates $(x, y)$ centered at $q$ as

$$
d g=\omega(r) d x d y
$$

Since

$$
\frac{d \nu_{q}}{d g}=\frac{\varphi_{\sigma}(r)}{\omega(r)}
$$

and the hyperbolic distance (i.e. the distance with respect to metric $h$ ) of a point $(x, y)$ from $(0,0)$ is dependent only on $r$, the above quotient is a function dependent only on the distance from $q$ with respect to the metric $g=\kappa^{-1} h$. In other words,

$$
d \nu_{q}(p)=\eta_{\sigma}\left(d_{g}(q, p)\right) d g(p) .
$$

Furthermore, since $g$ is a scalar multiple of the hyperbolic metric, the function $\eta_{\sigma}\left(d_{g}(q, p)\right)$ has the following property: if we denote the Laplacian in $g$ with respect to variables $q$ and $p$ by $\triangle_{g}^{q}$ and $\triangle_{g}^{p}$ respectively, then for any $\left(p_{0}, q_{0}\right) \in$ $\tilde{S} \times \tilde{S}$

$$
\left.\triangle_{g}^{q} \eta_{\sigma}\left(d_{g}(q, p)\right)\right|_{(p, q)=\left(p_{0}, q_{0}\right)}=\left.\triangle_{g}^{p} \eta_{\sigma}\left(d_{g}(q, p)\right)\right|_{(p, q)=\left(p_{0}, q_{0}\right)} .
$$

Indeed, if $I$ is an isometry of the hyperbolic plane switching the points $p_{0}$ and $q_{0}$, then

$$
\begin{aligned}
\left.\triangle_{g}^{q} \eta_{\sigma}\left(d_{g}(q, p)\right)\right|_{(p, q)=\left(p_{0}, q_{0}\right)} & =\left.\triangle_{g}^{q} \eta_{\sigma}\left(d_{g}(I q, I p)\right)\right|_{(p, q)=\left(p_{0}, q_{0}\right)} \\
& =\left.\triangle_{g}^{q} \eta_{\sigma}\left(d_{g}(q, p)\right)\right|_{(p, q)=\left(I p_{0}, I q_{0}\right)} \\
& =\left.\triangle_{g}^{q} \eta_{\sigma}\left(d_{g}(q, p)\right)\right|_{(p, q)=\left(q_{0}, p_{0}\right)} \\
& =\left.\triangle_{g}^{p} \eta_{\sigma}\left(d_{g}(p, q)\right)\right|_{(p, q)=\left(p_{0}, q_{0}\right)} \\
& =\left.\triangle_{g}^{p} \eta_{\sigma}\left(d_{g}(q, p)\right)\right|_{(p, q)=\left(p_{0}, q_{0}\right)} .
\end{aligned}
$$

We define a smooth function $f^{\sigma}: \tilde{S} \rightarrow[0, \infty)$ by setting

$$
f^{\sigma}(q)=\exp \left(\int_{\tilde{S}} \log f d \nu_{q}\right)
$$


and a smooth metric $G^{\sigma}$ by setting

$$
G^{\sigma}:=f^{\sigma} g
$$

Since the metric $g$, the function $f$ and the measure $d \nu_{q}$ are all $\rho$-invariant, so is $G^{\sigma}$. Jensen's inequality implies

$$
f^{\sigma}(q)=\exp \left(\int_{\tilde{S}} \log f(p) d \nu_{q}(p)\right) \leq \int_{\tilde{S}} f(p) d \nu_{q}(p)
$$

In the Poincaré coordinates $(x, y)$ centered at $q \in \tilde{S}$, we will write

$$
\begin{gathered}
G=\lambda\left(d x^{2}+d y^{2}\right), \quad \lambda=f \omega, \\
G^{\sigma}=\lambda^{\sigma}\left(d x^{2}+d y^{2}\right), \quad \lambda^{\sigma}=f^{\sigma} \omega
\end{gathered}
$$

and the function $p \mapsto \eta_{\sigma}\left(d_{g}(p, q)\right)$ as $\eta(r)$. With the above notation, we compute in the Poincaré coordinates $(x, y)$ centered at $q$ to obtain

$$
\begin{aligned}
& \int_{\tilde{S}} \log f(x, y) \triangle \eta(r) d x d y+2 \kappa \\
& =\int_{\tilde{S}} \log \lambda(x, y) \triangle \eta(r) d x d y-\int_{\tilde{S}} \log \omega(r) \triangle \eta(r) d x d y+2 \kappa \quad(\text { by }(9)) \\
& \left.=\int_{\tilde{S}} \log \lambda(x, y) \triangle \eta(r) d x d y-2 \kappa \int_{\tilde{S}} \eta(r) \omega(r) d x d y+2 \kappa \quad \text { (by }(4)\right) \\
& \left.=\int_{\tilde{S}} \log \lambda(x, y) \triangle \eta(r) d x d y \quad \text { (by }(5) \text { and }(6)\right) \\
& \left.\geq 2 \int_{\tilde{S}} \lambda(x, y) \eta(r) d x d y \quad \text { (by Theorem } 13\right) \\
& \left.=2 \int_{\tilde{S}} f(x, y) \eta(r) \omega(r) d x d y \quad \text { (by }(9)\right) .
\end{aligned}
$$

Since

$$
\triangle_{g}^{p} \eta_{\sigma}\left(d_{g}(q, p)\right) d g(p)=\frac{1}{\omega(r)} \triangle \eta_{\sigma}(r) \omega(r) d x d y=\triangle \eta_{\sigma}(r) d x d y
$$

we conclude

$$
\int_{\tilde{S}} \log f(p) \triangle_{g}^{p} \eta_{\sigma}\left(d_{g}(q, p)\right) d g(p)+2 \kappa \geq 2 \int_{\tilde{S}} f \eta_{\sigma}\left(d_{g}(q, p)\right) d g(p) .
$$


To obtain the Gaussian curvature of $G^{\sigma}$, we compute

$$
\begin{aligned}
& \left.\frac{1}{\omega(0,0)} \triangle \log \lambda^{\sigma}\right|_{(0,0)} \\
& =\left.\frac{1}{\omega} \triangle \log f^{\sigma}\right|_{(0,0)}+\left.\frac{1}{\omega} \triangle \log \omega\right|_{(0,0)} \\
& =\triangle_{g}^{q}\left(\int_{\tilde{S}} \log f(p) d \nu_{q}(p)\right)+2 \kappa \\
& =\int_{\tilde{S}} \log f(p) \triangle_{g}^{q} \eta_{\sigma}\left(d_{g}(q, p)\right) d g(p)+2 \kappa \\
& =\int_{\tilde{S}} \log f(p) \triangle_{g}^{p} \eta_{\sigma}\left(d_{g}(q, p)\right) d g(p)+2 \kappa \\
& \geq 2 \int_{\tilde{S}} f(p) \eta_{\sigma}\left(d_{g}(q, p)\right) d g(p) \quad(\text { by }(11)) \\
& =2 \int_{\tilde{S}} f(p) d \nu_{q}(p) \quad(\text { by }(6)) \\
& \geq 2 f^{\sigma}(0,0) . \quad(\text { by }(8)) .
\end{aligned}
$$

In other words,

$$
K^{\sigma}(q)=-\left.\frac{1}{2 \lambda^{\sigma}(0,0)} \triangle \log \lambda^{\sigma}\right|_{(0,0)} \leq-1
$$

which proves $(i)$.

The set $E=\left\{q \in \tilde{S}: K^{\sigma}(q)=-1\right\}$ is closed by the continuity of $K^{\sigma}$. We now also prove $E$ is open. Assume $E$ is non-empty and let $q \in E$ and identify it with $(0,0)$ via the Poincaré coordinates centered at $q$. Since $K^{\sigma}(q)=-1$, we must have equalities in the string of inequalities (12). In particular, we have an equality in Jensen's inequality (8) which implies that $\log f$ must be a constant, say $c$ in the support of $\eta_{\sigma}$. Thus, $\lambda=e^{c} \omega$ in the support of $\eta_{\sigma}$. Furthermore,

$$
\int_{\tilde{S}} \log \lambda(x, y) \triangle \eta(r) d x d y=2 \int_{\tilde{S}} \lambda(x, y) \eta(x, y) d x d y
$$

which then implies $e^{c}=\kappa$. Plugging in $f=\kappa$ in the definition (8) of $f^{\sigma}$, we obtain $f^{\sigma}=\kappa$. In other words, $G=G^{\sigma}=\kappa \omega\left(d x^{2}+d y^{2}\right)=h$ in the support of $\eta_{\sigma}$. Thus, the support of $\eta_{\sigma}$ is contained in $E$ which proves $E$ is open. Since $E$ is open and closed and non-empty by assumption, $E=\tilde{S}$. Since we 
have shown that $G=G^{\sigma}=h$ in a neighborhood of every point in $E$, this proves $(i i)$.

We are left to prove $($ iii $)$. For a non-negative $\varphi \in C_{c}^{\infty}\left(\mathbb{R}^{2}\right)$,

$$
\begin{aligned}
-\int_{\tilde{S}} \nabla \varphi \cdot \nabla \log f d x d y & =\int_{\tilde{S}}(\triangle \varphi) \log f d x d y \\
& =\int_{\tilde{S}}(\triangle \varphi) \log \lambda d x d y-\int_{\tilde{S}}(\triangle \varphi) \log \omega d x d y \\
& \geq 2 \int_{\tilde{S}} \varphi(\lambda-\kappa \omega) d x d y,
\end{aligned}
$$

where the last inequality follows from Theorem 13 and equation (4) after integrating by parts.

For $\tau \in(0, \sigma]$, we apply the above inequality with a test function $\varphi$ which approximates the characteristic function of $\mathbb{B}_{\tau}(0)$. More precisely, let $\varphi=\varphi(r)$ be a radially symmetric smooth function on $\mathbb{B}_{\tau}(0)$, non-increasing in $r$ such that $\varphi=1$ on $\mathbb{B}_{\tau-2 \varepsilon}(0)$ and $\varphi=0$ on $\mathbb{B}_{\tau}(0) \backslash \mathbb{B}_{\tau-\varepsilon}(0)$. Letting $\varepsilon \rightarrow 0$, we obtain

$$
\int_{\partial \mathbb{B}_{\tau}(0)} \frac{\partial}{\partial r} \log f d s \geq 2 \int_{\mathbb{B}_{\tau}(0)} \lambda-\kappa \omega d x d y \text { for a.e. } \tau \in(0, \sigma]
$$

where $d s$ is the line element of $\partial \mathbb{B}_{r}(0)$. Using polar coordinates $(r, \theta)$,

$$
\begin{aligned}
\left.\frac{\partial}{\partial r}\left(\int_{0}^{2 \pi} \log f(r, \theta) d \theta\right)\right|_{r=\tau} & =\left.\int_{0}^{2 \pi} \frac{\partial}{\partial r} \log f(r, \theta)\right|_{r=\tau} d \theta \\
& =\left.\frac{1}{\tau} \int_{0}^{2 \pi} \frac{\partial}{\partial r} \log f(r, \theta)\right|_{r=\tau} \tau d \theta \\
& \geq \frac{2}{\tau} \int_{0}^{2 \pi} \int_{0}^{\tau} \lambda-\kappa \omega r d r d \theta
\end{aligned}
$$

Thus, for $\rho, t \in(0, \sigma)$ and $t<\rho$,

$$
\begin{aligned}
& \int_{0}^{2 \pi} \log f(\rho, \theta) d \theta-\int_{0}^{2 \pi} \log f(t, \theta) d \theta \\
& \quad=\left.\int_{t}^{\rho} \frac{\partial}{\partial r}\left(\int_{0}^{2 \pi} \log f(r, \theta) d \theta\right)\right|_{r=\tau} d \tau \\
& \quad \geq \int_{t}^{\rho} \frac{2}{\tau} \int_{\mathbb{B}_{\tau}(0)}(\lambda-\kappa \omega) d x d y d \tau
\end{aligned}
$$


Multiply the above inequality by $t$ and integrate with respect to $t$ over interval $[0, s]$ where $s<\rho$ to obtain

$$
\begin{aligned}
& \frac{s^{2}}{2} \int_{0}^{2 \pi} \log f(\rho, \theta) d \theta-\int_{\mathbb{B}_{s}(0)} \log f(t, \theta) d x d y \\
& \quad=\int_{0}^{s} t \int_{0}^{2 \pi} \log f(r, \theta) d \theta d t-\int_{0}^{s} \int_{0}^{2 \pi} \log f(t, \theta) t d \theta d t \\
& \quad \geq \int_{0}^{s} t \int_{t}^{\rho} \frac{2}{\tau} \int_{\mathbb{B}_{\tau}(0)}(\lambda-\kappa \omega) d x d y d \tau d t .
\end{aligned}
$$

Since we only need to prove the inequality of $(i i i)$ for a.e. $q \in \tilde{S}$, we can assume without the loss of generality that $q$ is a Lebesgue point for the integrable function $\log \lambda$. Thus dividing the above inequality by $\pi s^{2}$ and letting $s \rightarrow 0$, we obtain

$$
\begin{aligned}
& \frac{1}{2 \pi} \int_{0}^{2 \pi} \log f(r, \theta) d \theta-\log f(q) \\
& \geq \lim _{s \rightarrow 0} \frac{1}{\pi s^{2}} \int_{0}^{s} t \int_{t}^{\rho} \frac{2}{\tau} \int_{\mathbb{B}_{\tau}(0)}(\lambda-\kappa \omega) d x d y d \tau d t \\
& =\lim _{s \rightarrow 0} \frac{1}{\pi s^{2}} \int_{0}^{s} t \int_{0}^{\rho} \frac{2}{\tau} \int_{\mathbb{B}_{\tau}(0)}(\lambda-\kappa \omega) d x d y d \tau d t \\
& \quad-\lim _{s \rightarrow 0} \frac{1}{\pi s^{2}} \int_{0}^{s} t \int_{0}^{t} \frac{2}{\tau} \int_{\mathbb{B}_{\tau}(0)}(\lambda-\kappa \omega) d x d y d \tau d t \\
& =\frac{1}{2 \pi} \int_{0}^{\rho} \frac{2}{\tau} \int_{\mathbb{B}_{\tau}(0)}(\lambda-\kappa \omega) d x d y d \tau \\
& \quad-\lim _{s \rightarrow 0} \frac{1}{\pi s^{2}} \int_{0}^{s} t \int_{0}^{t} \frac{2}{\tau} \int_{\mathbb{B}_{\tau}(0)}(\lambda-\kappa \omega) d x d y d \tau d t .
\end{aligned}
$$

Since $|\lambda-\kappa \omega|$ is a bounded function in $\mathbb{B}_{\sigma}(0)$, we conclude that given $\epsilon>0$, there exists $t_{0}>0$ sufficiently small such that

$$
t \in\left(0, t_{0}\right) \Rightarrow \int_{0}^{t} \frac{2}{\tau} \int_{\mathbb{B}_{\tau}(0)}(\lambda-\kappa \omega) d x d y d \tau<\epsilon .
$$

Thus

$$
s \in\left(0, t_{0}\right) \Rightarrow \frac{1}{\pi s^{2}} \int_{0}^{s} t \int_{0}^{t} \frac{2}{\tau} \int_{\mathbb{B}_{\tau}(0)}(\lambda-\kappa \omega) d x d y d \tau d t \leq \frac{\epsilon}{2 \pi} .
$$


We can now conclude that the limit that appears in (13) is equal to 0, and therefore

$$
\int_{0}^{2 \pi} \log f(r, \theta) d \theta \geq 2 \pi \log f(q)+\int_{0}^{\rho} \frac{2}{\tau} \int_{\mathbb{B}_{\tau}(0)}(\lambda-\kappa \omega) d x d y d \tau
$$

By the definition of $f^{\sigma}$, we have

$$
f^{\sigma}(q)=\exp \int_{0}^{\sigma}\left(\int_{0}^{2 \pi} \log f(r, \theta) d \theta\right) \eta(r) \omega(r) r d r .
$$

Combining the above,

$$
f^{\sigma}(q) \geq f(q) \exp \int_{0}^{\sigma}\left(\int_{0}^{\rho} \frac{1}{\tau} \int_{\mathbb{B}_{\tau}(0)}(\lambda-\kappa \omega) d x d y d \tau\right) \eta(r) \omega(r) r d r .
$$

The function

$$
\psi: \tilde{S} \rightarrow[0, \infty), \quad \psi(q):=\int_{0}^{\sigma}\left(\int_{0}^{\rho} \frac{1}{\tau} \int_{\mathbb{B}_{\tau}(0)} \lambda d x d y d \tau\right) \eta(r) \omega(r) r d r
$$

is continuous by Theorem $13(i)$. Furthermore, $\psi(q)>0$ for all $q \in \tilde{S}$ since Theorem 13 (iv) implies

$$
\int_{\mathbb{B}_{\tau}(0)} \lambda d x d y>0, \quad \forall \tau>0
$$

Since $\psi$ is also equivariant, this implies a positive lower bound for $\psi$; i.e. $\min _{q \in \tilde{S}} \psi(q)>0$. Moreover, the function

$$
q \mapsto \int_{0}^{\sigma}\left(\int_{0}^{\rho} \frac{1}{\tau} \int_{\mathbb{B}_{\tau}(0)} \omega d x d y d \tau\right) \eta(r) \omega(r) r d r
$$

is constant. Thus, we can choose $\kappa>0$ sufficiently small such that

$$
\int_{0}^{\sigma}\left(\int_{0}^{\rho} \int_{\mathbb{B}_{\tau}(0)}(\lambda-\kappa \omega) d x d y d \tau\right) \eta(r) \omega(r) r d r \geq 0 .
$$

Thus, (14) implies (iii). Q.E.D. 


\section{Proof of Theorem 1 and Theorem 2}

Given a convex cocompact representation $\rho: \pi_{1}(S) \rightarrow \operatorname{Isom}(X)$, let $h \in \mathcal{T}$ be the hyperbolic metric and $\tilde{u}: \mathbb{H}^{2}=(\tilde{S}, h) \rightarrow X$ be the $\rho$-equivariant conformal harmonic map of Proposition 12. Let $G$ be as in Definition 14 and $G^{\sigma}$ be the smooth metric of Lemma 16. In local coordinates, we write

$$
G=\lambda\left(d x^{2}+d y^{2}\right), \quad G^{\sigma}=\lambda^{\sigma}\left(d x^{2}+d y^{2}\right) \quad \text { and } \quad h=\omega\left(d x^{2}+d y^{2}\right) .
$$

Let $d_{h}$ and $d_{G^{\sigma}}$ be the distance function induced from the metrics $h$ and $G^{\sigma}$ respectively on $\tilde{S}$. We can also define the distance function $d_{G}$ induced from $G$. Indeed, since $\lambda$ is locally a $H^{1}$ function (cf. Theorem $16(i)$ ), we can define a length of a smooth curve $c:[0,1] \rightarrow \widetilde{S}$ by letting

$$
\operatorname{length}(c)=\int_{0}^{1} \sqrt{\lambda}\left|c^{\prime}(t)\right| d t
$$

Then

$$
d_{G}(x, y)=\inf _{c \in \mathcal{C}} \operatorname{length}(c)
$$

where $\mathcal{C}$ consists of all smooth curves $c:[0,1] \rightarrow \tilde{S}$ with $c(0)=x$ and $c(1)=y$.

Proof of Theorem 2. We define an equivariant smooth function

$$
f^{\sigma}: \tilde{S} \rightarrow(0, \infty), \quad f^{\sigma}=\frac{G^{\sigma}}{h} .
$$

In local coordinates, we have $f^{\sigma}=\frac{\lambda^{\sigma}}{\omega}$. Let $p_{0}$ be the point at which $f^{\sigma}$ achieves a maximum. Thus, $\triangle \log f^{\sigma}\left(p_{0}\right) \leq 0$. If $f^{\sigma}\left(p_{0}\right)=\frac{\lambda^{\sigma}\left(p_{0}\right)}{\omega\left(p_{0}\right)}>1$, then Lemma $16(i)$ says

$$
\triangle \log f^{\sigma}\left(p_{0}\right)=\triangle \log \lambda^{\sigma}\left(p_{0}\right)-\triangle \log \omega\left(p_{0}\right) \geq 2 \lambda^{\sigma}\left(p_{0}\right)-2 \omega\left(p_{0}\right)>0,
$$

a contradiction. Thus, combined with Lemma 16 (iii), we conclude

$$
\lambda \leq \lambda^{\sigma} \leq \omega .
$$

in every coordinate neighborhood. Combining this inequality with the fact that $G$ is the pullback of $\tilde{u}$, we obtain

$$
d(\tilde{u}(p), \tilde{u}(q)) \leq d_{G}(p, q) \leq d_{G^{\sigma}}(p, q) \leq d_{h}(p, q), \quad \forall p, q \in \tilde{S}
$$


which proves $\tilde{u}$ is a contracting map.

We next prove that $\tilde{u}$ is strictly contracting unless $\tilde{u}$ is a totally geodesic map and an isometric embedding of $\mathbb{H}^{2}$. Indeed, if there exists $p, q$ such that $d(\tilde{u}(p), \tilde{u}(q))=d_{h}(p, q)$, then there exists $p_{0} \in \tilde{S}$ such that $f^{\sigma}\left(p_{0}\right)=\frac{\lambda^{\sigma}\left(p_{0}\right)}{\omega\left(p_{0}\right)}=$ 1. Since we have already shown $f^{\sigma} \leq 1$, this implies that the function $f^{\sigma}$ achieves a maximum at $p_{0}$. Thus,

$$
0 \geq \triangle \log f^{\sigma}\left(p_{0}\right)=\triangle \log \lambda^{\sigma}\left(p_{0}\right)-\triangle \log \omega\left(p_{0}\right) \geq 2 \lambda^{\sigma}\left(p_{0}\right)-2 \omega\left(p_{0}\right)=0 .
$$

This in turn implies

$$
K^{\sigma}\left(p_{0}\right)=-\frac{1}{2 \lambda^{\sigma}\left(p_{0}\right)} \triangle \log \lambda^{\sigma}\left(p_{0}\right)=-\frac{1}{2 \omega\left(p_{0}\right)} \triangle \log \omega\left(p_{0}\right)=-1 .
$$

By Lemma 16 (ii), we conclude that $G=h$.

We now show that the equality $G=h$ implies that $\tilde{u}$ is a totally geodesic map and an isometric embedding of $\mathbb{H}^{2}$. For any $p \in \tilde{S}$, identify $p=0$ via normal coordinates and let $(r, \theta)$ be polar coordinates. For $R>0$, define $\phi: B_{R}(p) \rightarrow X$ by setting

$$
\phi(r, \theta)=(1-r) \tilde{u}(p)+r \tilde{u}(1, \theta)
$$

where, using a common notation in NPC geometry, the sum on the right hand side denotes the geodesic interpolation. In other words, $\phi$ maps geodesics emanating from $p$ to geodesics emanating from $\tilde{u}(p)$. Therefore,

$$
\left|\frac{\partial \phi}{\partial r}\right|^{2}=d^{2}(\tilde{u}(1, \theta), \tilde{u}(p)) \leq d_{G}^{2}((1, \theta), p) .
$$

We next claim that

$$
\left|\frac{\partial \tilde{u}}{\partial r}\right|^{2}(r, \theta)=\lim _{\epsilon \rightarrow 0} \frac{d_{G}^{2}((r, \theta),(r+\epsilon, \theta))}{\epsilon^{2}}
$$

Indeed, since $G$ is equal to the hyperbolic metric $h$ and $(r, \theta)$ are polar coordinates for $h$

$$
\begin{aligned}
\lim _{\epsilon \rightarrow 0} \frac{d_{G}^{2}((r, \theta),(r+\epsilon, \theta))}{\epsilon^{2}} & =\lim _{\epsilon \rightarrow 0}\left(\frac{1}{\epsilon} \int_{r}^{r+\epsilon} \sqrt{\lambda}(s, \theta) d s\right)^{2} \\
& =\lambda(r, \theta)=\left|\frac{\partial \tilde{u}}{\partial r}\right|^{2}(r, \theta)
\end{aligned}
$$


the last equality being because $\tilde{u}$ is conformal. On the other hand, again since $(r, \theta)$ are polar coordinates for $h=G$

$$
\lim _{\epsilon \rightarrow 0} \frac{d_{G}^{2}((r, \theta),(r+\epsilon, \theta))}{\epsilon^{2}}=d_{G}^{2}((1, \theta), p) .
$$

Combining equalities (18), (19) and (20) we obtain

$$
\left|\frac{\partial \phi}{\partial r}\right|^{2}(r, \theta) \leq\left|\frac{\partial \tilde{u}}{\partial r}\right|^{2}(r, \theta) .
$$

Furthermore,

$$
d\left(\phi\left(1, \theta_{1}\right), \phi\left(1, \theta_{2}\right)\right)=d\left(u\left(1, \theta_{1}\right), u\left(1, \theta_{2}\right)\right) \leq d_{G}\left(\left(1, \theta_{1}\right),\left(1, \theta_{2}\right)\right) .
$$

Since we have shown that $G$ is the hyperbolic metic, the CAT(-1) condition implies that

$$
d\left(\phi\left(r, \theta_{1}\right), \phi\left(r, \theta_{2}\right)\right) \leq d_{G}\left(\left(r, \theta_{1}\right),\left(r, \theta_{2}\right)\right) .
$$

Thus,

$$
\begin{aligned}
\left|\frac{\partial \phi}{\partial \theta}\right|^{2}(r, \theta) & =\lim _{\epsilon \rightarrow 0} \frac{d^{2}(\phi(r, \theta), \phi(r, \theta+\epsilon))}{\epsilon^{2}} \\
& \leq \lim _{\epsilon \rightarrow 0} \frac{d_{G}^{2}((r, \theta),(r, \theta+\epsilon))}{\epsilon^{2}}=\left|\frac{\partial \tilde{u}}{\partial \theta}\right|^{2}(r, \theta) .
\end{aligned}
$$

Notice that the derivation of the last equality is similar to that of (19). Thus (21) and (22) imply that $E^{\phi} \leq E^{\tilde{u}}$, but since $\tilde{u}$ is energy minimizing $\phi=\tilde{u}$. Therefore, $\tilde{u}$ maps radial lines emanating from $p$ to geodesics. Since $p$ is an arbitrary point in $\tilde{S}$, this proves $\tilde{u}$ is a totally geodesic map and an isometric embedding of $\mathbb{H}^{2}$. Q.E.D.

Proof of Theorem 1. For a discrete subgroup $\Gamma$ of $\operatorname{Isom}(X)$, the critical exponent for the Poincaré series

$$
P^{s}(p, q)=\sum_{\gamma \in \Gamma} e^{-s d(p, \gamma q)}
$$

for $s>0$ and $p, q \in X$ is (cf. [Co] Proposition 5.3)

$$
\delta_{d}(\Gamma)=\lim _{R \rightarrow \infty} \frac{\log N_{\Gamma}^{d}(R, p)}{R}, \quad N_{\Gamma}^{d}(R, p)=\#\{\gamma \in \Gamma: d(\gamma p, p) \leq R\} .
$$


Letting $\Gamma=\rho\left(\pi_{1}(S)\right)$ and noting (17), we have

$$
N_{\pi_{1}(S)}^{d_{h}}(R, x) \leq N_{\pi_{1}(S)}^{d_{G^{\sigma}}}(R, x) \leq N_{\pi_{1}(S)}^{d_{G}}(R, x) \leq N_{\Gamma}^{d}(R, \tilde{u}(x)) .
$$

Take logarithm, divide by $R$ and let $R \rightarrow \infty$ for both sides of the above inequality. On the left hand side, we obtain $\delta_{d_{h}}\left(\pi_{1}(S)\right)=1$. On the right hand side, we obtain $\operatorname{dim}_{\mathcal{H}}(\Lambda)$; indeed, the critical exponent of any convex cocompact action on a CAT $(-1)$ space coincides with the Hausdorff dimension of the limit set computed with respect to the Gromov metric(cf. [Co]). In summary, we have shown

$$
1=\delta_{h}\left(\pi_{1}(S)\right) \leq \delta_{G^{\sigma}}\left(\pi_{1}(S)\right) \leq \delta_{G}\left(\pi_{1}(S)\right) \leq \delta_{d}(\Gamma)=\operatorname{dim}_{\mathcal{H}}(\Lambda) .
$$

(Note that this implies the assertion of Corollary 4). Thus, the assumption that $\operatorname{dim}_{\mathcal{H}}(\Lambda)=1$ implies $\delta_{G^{\sigma}}\left(\pi_{1}(S)\right)=1$.

By Lemma $16(i), G^{\sigma}$ is negatively curved, therefore [Man1] implies that $\delta_{G^{\sigma}}\left(\pi_{1}(S)\right)$ is equal to the topological entropy of the geodesic flow, which

dominates the measure theoretic entropy by the variational principle. Therefore, [Man2] implies that

$$
1=\delta_{G^{\sigma}}\left(\pi_{1}(S)\right) \geq \frac{1}{\operatorname{Vol}_{G^{\sigma}}(S)} \int_{S} \sqrt{-K^{\sigma}} d \operatorname{Vol}_{G^{\sigma}}
$$

where $K^{\sigma}$ is the Gauss curvature and $\operatorname{Vol}_{G^{\sigma}}$ is the volume form with respect to the smooth metric $G^{\sigma}$. Lemma $16(i)$ establishes that $-K^{\sigma} \geq 1$, therefore the above inequality implies that $K^{\sigma} \equiv-1$. Thus, $G=h$ by Lemma 16 (ii). As in the proof of Theorem 2, we conclude that $\tilde{u}$ is a totally geodesic map and an isometric embedding of $\mathbb{H}^{2}$. Q.E.D.

\section{References}

[BK] M. Bonk and B. Kleiner. Ridigity for quasi-Fuchsian actions on negatively curved spaces. International Mathematical Research Notices (2004) 3309-3316.

[Bou] M. Bourdon. Structure conforme au bord et flot géodésique d'un CAT(-1) espace. Enseign. Math. 41 (1995) 63 -102. 
[Bow] R. Bowen. Hausdorff dimension of quasicircles. Inst. Hautes tudes Sci. Publ. Math. No. 50 (1979), 11-25.

[Co] M. Coornaert. Mesures de Patterson-Sullivan sur le bord d'un espace hyperbolique au sens de Gromov. Pacific J. Math 159 (1993) 241-270.

[DT] B. Deroin and N. Tholozan. Dominating surface group representations by Fuchsian ones. International Mathematics Research Notices 2016 (2016) 4145-4166.

[GW] W. Goldman and R. Wentworth. Energy of twisted harmonic maps of Riemann surfaces. In the tradition of Ahlfors-Bers. IV, 45-61, Contemp. Math., 432, Amer. Math. Soc., Providence, RI, 2007.

[Kn] G. Knieper. On the asymptotic geometry of nonpositively curved manifolds. GAFA 7 (1997) 755-782.

[KS1] N. Korevaar and R. Schoen. Sobolev spaces and harmonic maps into metric space targets. Comm. Anal. Geom. 1 (1993) 561-659.

[KS2] N. Korevaar and R. Schoen. Global existence theorem for harmonic maps to non-locally compact spaces. Comm. Anal. Geom. 5 (1997) 333-387.

[Man1] A. Manning. Topological entropy for geodesic flows. Ann. of Math. 110 (1979) 567-573.

[Man2] A. Manning. Curvature bounds for the entropy of the geodesic flow on a surface. J. London Math. Soc. 24 (1981) 351-357.

[Mes] C. Mese. The curvature of minimal surfaces in singular spaces. Comm. Anal. Geom. 9 (2001) 3-34.

[San] A. Sanders. Entropy, minimal surfaces and negatively curved manifolds. Preprint.

[SY] R. Schoen and S.T. Yau. Existence of Incompressible Minimal Surfaces and the Topology of Three Dimensional Manifolds with NonNegative Scalar Curvature. Annals of Mathematics 110 (1979) 127142. 